\title{
Spiroergometrie bei bettlägerigen Patienten mit schwergradiger COB
}

\author{
W. Galetke \\ W. Randerath \\ M. Pfeiffer \\ F. Feldmeyer \\ K.-H. Rühle
}

\author{
Spiroergometry in Patients with Severe Chronic Obstructive Pulmonary \\ Disease Confined to Bed
}

\section{Zusammenfassung}

Hintergrund: Körperliches Training wird für Patienten mit einer chronisch-obstruktiven Bronchitis (COB) zur Steigerung der Belastbarkeit empfohlen. Wir untersuchten bei bettlägerigen Patienten mit schwergradiger COB den Einfluss einer Spiroergometrie im Bett auf atemphysiologische Parameter. Methode: Bei neun bettlägerigen Patienten mit schwergradiger COB (FEV1.0 $0,94 \pm 0,18 \mathrm{l}$, IVC $2,3 \pm 0,81$, Raw $0,91 \pm 0,13 \mathrm{kPa} / \mathrm{l} / \mathrm{s}$ ) wurden in Ruhe, bei passiver Bewegung mit $30 \mathrm{U} / \mathrm{min}$, zusätzlicher aktiver Tretarbeit sowie unter maximal möglicher Umdrehungszahl die Parameter Sauerstoffaufnahme $\mathrm{VO}_{2}$, Atemfrequenz $\mathrm{BF}$ und Atemminutenvolumen VE gemessen. Als Vergleichskollektiv dienten sechs Gesunde, bei denen die Werte in Ruhe und unter passiver Bewegung bestimmt wurden. Ergebnisse: Die COB-Patienten erreichten bei maximaler Tretarbeit ein $\mathrm{VO}_{2}$ peak von $618 \pm 177 \mathrm{ml} / \mathrm{min}$, eine $\mathrm{BF}$ von $26 \pm 7,2 / \mathrm{min}$ und ein VE $\max$ von $24,1 \pm 5 \mathrm{l} / \mathrm{min}$. In Ruhe betrug die $\mathrm{VO}_{2} 311 \pm 56 \mathrm{ml} / \mathrm{min}(53 \%$ des $\mathrm{VO}_{2}$ peak), die BF $17,6 \pm 3,1 / \mathrm{min}$ und das $\mathrm{VE} 13,3 \pm 2,7 \mathrm{ml} / \mathrm{min}$ ( $55 \%$ des VE max). Unter passiver Bewegung mit $30 \mathrm{U} / \mathrm{min}$ zeigte sich eine Steigerung der $\mathrm{VO}_{2}$ auf $369 \pm 88\left(62 \% \mathrm{VO}_{2}\right.$ peak), der $\mathrm{BF}$ auf $19 \pm 5,3$ und des VE auf 16,4 $\pm 4,1$ (68\% VE max). Die Gesunden lagen in Ruhe mit ihrer $\mathrm{VO}_{2}$ bei $377,5 \pm 38$, mit der $\mathrm{BF}$ bei $14 \pm 2,1$ und mit dem VE bei 11,1 $\pm 1,3$; unter Passiv-Bewegung fielen $\mathrm{VO}_{2}$ auf $336 \pm 27$, BF auf $12 \pm 2,4$ und VE auf $9,1 \pm 1$ ab. Schlussfolgerungen: Eine Bettergometrie steigert bei bettlägerigen COB-Patienten sowohl bei rein passiver als auch zusätzlicher aktiver Bewegung Sauerstoffaufnahme, Atemfrequenz und Atemminutenvolumen. Damit kann bei diesen Patienten ein körperliches Training im Bett durchgeführt werden.

\section{Abstract}

Background: Exercise training is recommended for patients with severe chronic obstructive pulmonary disease (COPD) to improve the endurance capacity. While many patients confined to bed are not able to run exercise training, we investigated the influence of a bedside passive-ergometry on ventilation in patients with severe COPD. Methods: In nine patients with severe COPD confined to bed (FEV1.0 0,94 $\pm 0,18$ l, IVC 2,3 $\pm 0,8$ l, Raw $0,91 \pm 0,13 \mathrm{kPa} / \mathrm{l} / \mathrm{s}$ ) we measured oxygen uptake $\mathrm{VO}_{2}$, breathing frequency $\mathrm{BF}$ and minute ventilation $\mathrm{VE}$ during rest, passive movement (30 revolutions per minute), additional active movement and maximal exercise. As a control group six healthy men were investigated during rest and passive movement. Results: During maximal exercise in COPD patients $\mathrm{VO}_{2}$ peak reached $618 \pm 177 \mathrm{ml} / \mathrm{min}, \mathrm{BF} 26 \pm 7,2 / \mathrm{min}$ and VE $\max 24,1 \pm 5 \mathrm{l} / \mathrm{min}$. In rest $\mathrm{VO}_{2}$ was $311 \pm 56 \mathrm{ml} / \mathrm{min}\left(53 \% \mathrm{VO}_{2}\right.$ peak), $\mathrm{BF} 17,6 \pm 3,1 / \mathrm{min}$ and $\mathrm{VE} 13,3 \pm 2,7 \mathrm{ml} / \mathrm{min}(55 \% \mathrm{VE} \max )$, while during passive movement $\mathrm{VO}_{2}$ was increased to $369 \pm 88\left(62 \% \mathrm{VO}_{2}\right.$ peak), $\mathrm{BF}$ to $19 \pm 5,3$ and $\mathrm{VE}$ to $16,4 \pm 4,1$ (68\% $\mathrm{VE}$ max). In contrast $\mathrm{VO}_{2}$ in control subjects dropped from $377,5 \pm 38$ in rest to $336 \pm 27 \mathrm{ml} /$ min during passive action, $\mathrm{BF}$ from $14 \pm 2,1$ to $12 \pm 2,4 / \mathrm{min}$ and VE from $11,1 \pm 1,3$ to $9,1 \pm 1 \mathrm{ml} / \mathrm{min}$. Conclusions: In patients with severe COPD oxygen uptake, breathing frequency and minute ventilation increased not only during active, but even during passive movement of a bedside ergometer. With this method an exercise training is possible even in COPD patients confined to bed. 
Patienten mit einer chronisch-obstruktiven Bronchitis (COB) leiden sehr häufig unter Belastungsdyspnoe, die ihr Korrelat in einer Limitation durch Ventilation und Gasaustausch bei spiroergometrischen Untersuchungen finden [1,2]. Daher umfassen die Therapieempfehlungen der COB neben medikamentösen und physikalischen Maßnahmen auch körperliches Training, dessen Effekte durch mehrere kontrollierte Studien gut belegt sind. Es zeigen sich eine Steigerung der maximalen körperlichen Belastbarkeit und der Gehstrecke ebenso wie eine Verbesserung der Lebensqualität und Abnahme krankheitsbedingter Symptome [3-9].

Bei Patienten mit schwerer COB ist ein körperliches Training aufgrund der oft erheblichen Muskelatrophie der unteren Extremitäten oder der bereits weitgehenden bzw. vollständigen Bettlägerigkeit mit den herkömmlichen Methoden wie Fahrrad- oder Laufband-Ergometer nicht mehr möglich. In der neurologischen und orthopädischen Rehabilitation kommen motorgetriebene Passiv-Ergometer zum Einsatz, die bei bettlägerigen Patienten passive und durch zusätzliche Mitarbeit des Patienten auch aktive Bewegungsübungen der unteren Extremitäten ermöglichen [10]. Die Anwendbarkeit einer derartigen Bewegungstherapie bei Patienten mit Erkrankungen der Lunge wurde bislang noch nicht in klinischen Studien überprüft.

Wir untersuchten daher den Einfluss der passiven Ergometrie und der zusätzlichen aktiven Tretarbeit auf atemphysiologische Parameter bei Gesunden und bettlägerigen Patienten mit schwergradiger $\mathrm{COB}$.

\section{Methodik}

Wir verwendeten das Gerät „MOTOmed letto“ (Fa. Reck, Betzenweiler) (Abb.1). Hierbei handelt es sich um ein motorbetriebenes Therapiegerät, bei dem im passiven Modus die Beine des im Bett befindlichen Patienten durch einen Elektromotor mit einer frei wählbaren Drehzahl zwischen 10 und 70 Umdrehungen/min bewegt werden. Im aktiven Modus besteht für den Patienten die Möglichkeit, schneller als die vorgegebene Umdrehungszahl zu treten. Die Pedalbelastung kann dabei elektronisch zwischen 5 und 25 Kilogramm reguliert werden.

Untersucht wurden neun weitgehend bettlägerige Patienten aus unserer Klinik mit schwergradiger chronisch-obstruktiver Bronchitis (COB) (Alter 69,2 \pm 9,5 Jahre, Gewicht 70,5 $\pm 13 \mathrm{~kg}$, Größe $167 \pm 10 \mathrm{~cm}$ ). Die Diagnose COB wurde aufgrund der Anamnese und der lungenfunktionsanalytischen Daten nach den Kriterien der ATS gestellt [11]. Der mittlere Sauerstoffpartialdruck $\mathrm{PaO}_{2}$ in der Patientengruppe betrug 56,8 $\pm 8,4 \mathrm{~mm} \mathrm{Hg}$ und der Kohlendioxidpartialdruck $\mathrm{PaCO}_{2} 44,8 \pm 14,8 \mathrm{~mm} \mathrm{Hg}$; zwei Patienten waren hyperkapnisch. Von fünf dieser Patienten lagen bodyplethysmographische Werte des letzten Jahres vor (FEV1.0 0,94 \pm 0,18 1, IVC $2,3 \pm 0,8$, Raw $0,91 \pm 0,13 \mathrm{kPa} / \mathrm{l} / \mathrm{s}$ ), wohingegen bei vier Patienten eine Lungenfunktionstestung aufgrund der Bettlägerigkeit nicht durchführbar war.

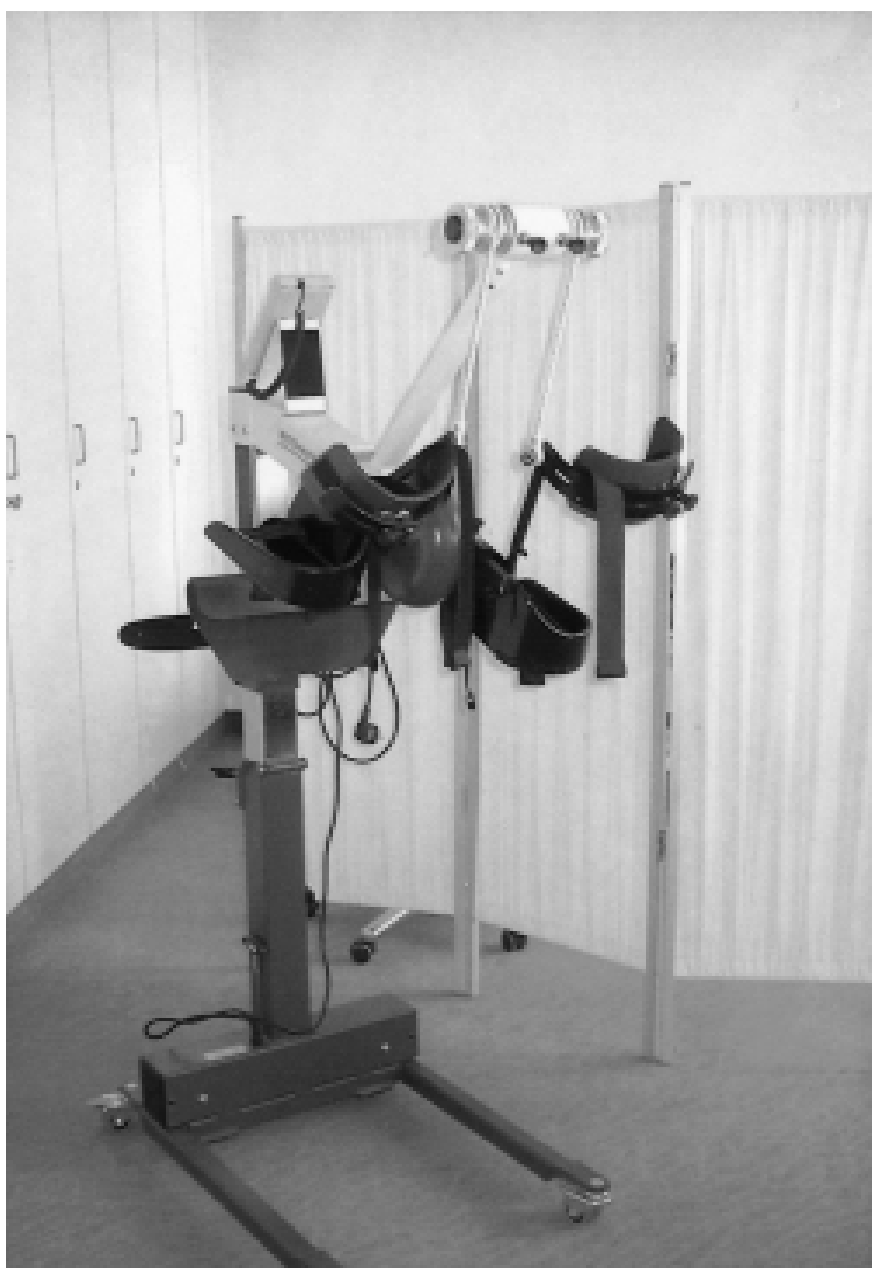

Abb. 1 Motorbetriebenes Therapiegerät „MOTOmed letto“ (Fa. Reck, Betzenweiler).

Die Patienten befanden sich zum Zeitpunkt der Untersuchung in einem klinisch stabilen Zustand ohne Hinweise für eine Infektexazerbation ihrer COB. Ausgeschlossen wurden Patienten mit einer akuten Myokardischämie oder kardialen Dekompensation, einer schlecht eingestellten arteriellen Hypertonie (RR $>$ 210/120 mm Hg), höhergradigen Rhythmusstörungen, relevanten Herzvitien oder anderen Erkrankungen, die eine Belastungsuntersuchung verboten. Alle Teilnehmer äußerten ihr Einverständnis zur Studie.

Als Vergleichskollektiv dienten sechs gesunde Mitarbeiter unserer Klinik (Alter $28 \pm 8,7$ Jahre, Gewicht $81,7 \pm 18,7 \mathrm{~kg}$, Größe $183 \pm 6 \mathrm{~cm})$.

Bei beiden Gruppen wurden zunächst spiroergometrische Messungen in Ruhe und bei passiver Bewegung mit 30 Umdrehungen (U/min) über fünf Minuten durchgeführt. Den Patienten mit COB wurde anschließend ein konstantes Drehmoment von $5 \mathrm{Nm}$ zugeschaltet und ein aktives Treten über die passiv vorgegebenen $30 \mathrm{U} / \mathrm{min}$ hinaus für weitere fünf Minuten ermöglicht. Abschließend wurden die Patienten unter Belassung des Widerstandes und der vorgegebenen Drehzahl aufgefordert, über zwei Minuten mit größtmöglicher Umdrehungszahl zu treten. 
Bei der Spiroergometrie (Oxycon Alpha, Fa. Jaeger, Würzburg) wurden als wesentliche Parameter die Sauerstoffaufnahme $\mathrm{VO}_{2}$, die Kohlendioxidabgabe $\mathrm{VCO}_{2}$, die Atemfrequenz $\mathrm{BF}$ und das Atemminutenvolumen VE in einer breath by breath-Analyse aufgezeichnet, wobei für jede Phase unter passiver und aktiver Belastung jeweils die gemittelten Werte über die Zeit verwendet wurden.

Zur Überprüfung der Unterschiede zwischen den einzelnen Belastungsstufen wurden als statistische Methoden die einfaktorielle Varianzanalyse mit Messwiederholungen, der FriedmanTest sowie der t-Test für gepaarte Stichproben verwandt. Bei einem $\mathrm{p}<0,05$ wurde der Unterschied als signifikant angesehen.

\section{Ergebnisse}

Die Sauerstoffaufnahme $\mathrm{VO}_{2}$ lag bei den COB-Patienten in Ruhe bei $311 \pm 56 \mathrm{ml} / \mathrm{min}$ und stieg unter alleiniger passiver Bewegung mit $30 \mathrm{U} / \mathrm{min}$ signifikant auf $369 \pm 88 \mathrm{ml} / \mathrm{min}(\mathrm{p}<0,02)$ an. Demgegenüber fiel $\mathrm{VO}_{2}$ bei der Kontrollgruppe von $378 \pm 38$ in Ruhe auf $336 \pm 27 \mathrm{ml} / \mathrm{min}$ bei passiver Bewegung ab (Abb. 2).

Bei der COB-Gruppe zeigte sich unter zusätzlichem aktiven Treten mit $5 \mathrm{Nm}$ eine weitere signifikante Steigerung der $\mathrm{VO}_{2}$ auf $467 \pm 116 \mathrm{ml} / \mathrm{min}(\mathrm{p}<0,005)$ und bei maximaler Tretkurbelarbeit eine $\mathrm{VO}_{2}$ von $618 \pm 117 \mathrm{ml} / \mathrm{min}\left(\mathrm{p}<0,001\right.$ gegenüber $\mathrm{VO}_{2}$ bei $30 \mathrm{U} / \mathrm{min}+5 \mathrm{Nm})($ Abb. 2).

Das Atemminutenvolumen VE stieg bei den COB-Patienten signifikant von 13,3 $\pm 2,71 /$ min in Ruhe auf $16,4 \pm 4,1(p<0,01)$ unter passiver und auf 19,1 $\pm 4,7 \mathrm{l} / \mathrm{min}$ ( $\mathrm{p}<0,01$ gegenüber $\mathrm{VE}$ passiv) unter zusätzlicher aktiver Bewegung an, während es bei maximaler Umdrehungszahl 24,1 $\pm 5 \mathrm{l} / \mathrm{min}$ betrug. Bei den Gesunden war ein Abfall von VE von 11,1 $\pm 1,3$ in Ruhe auf $9,1 \pm 1 \mathrm{l} / \mathrm{min}$ bei rein passiver Bewegung zu verzeichnen (Abb. 3).

Dementsprechend zeigte sich ein Anstieg der Atemfrequenz bei der COB-Gruppe von $17,6 \pm 3,1 / \mathrm{min}$ in Ruhe auf $19 \pm 5,3$ (n.s.) bei passiver bzw. auf 22,1 $\pm 6,2(p=0,01)$ bei aktiver Tretkurbelarbeit und auf 26/min $(p<0,001)$ unter maximal möglicher Umdrehungszahl. Bei der Kontrollgruppe fiel die Atemfrequenz von $14 \pm 2,1$ in Ruhe auf $12 \pm 2,4$ bei Passivbewegung ab (Abb. 4).

Die COB-Patienten nahmen bereits $53 \%$ ihres $\mathrm{VO}_{2}$ peak in Ruhe und $62 \%$ bzw. $72 \%$ ihres $\mathrm{VO}_{2}$ peak bei alleiniger passiver bzw. zusätzlicher aktiver Tretarbeit auf. Das Atemminutenvolumen in Ruhe betrug $55 \%$ des maximal erreichten Wertes, während es beim passiven Treten $68 \%$ und bei der aktiven Bewegung $79 \%$ von VE max erreichte (Abb. 5).

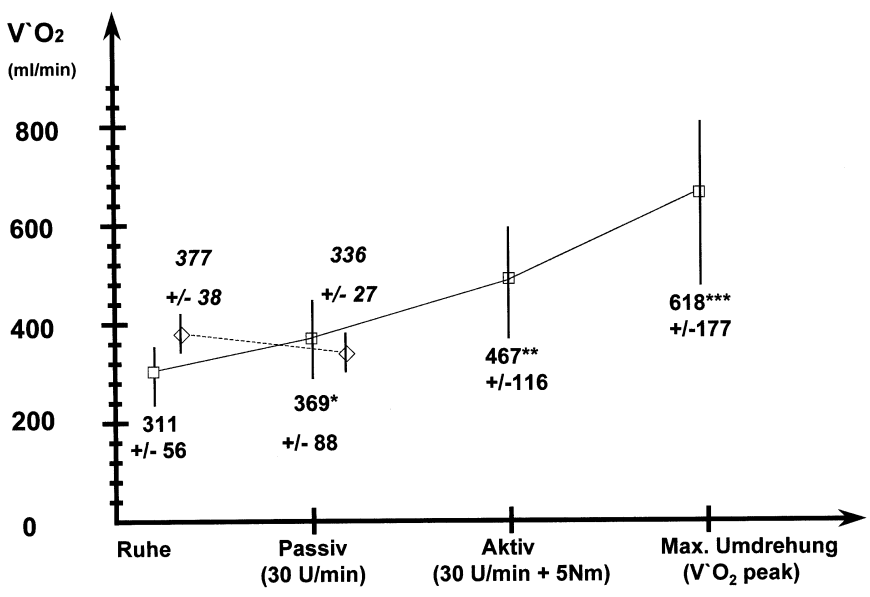

Abb. 2 Sauerstoffaufnahme $\dot{\mathrm{VO}}_{2}$ der COB-Patienten (Rechtecke) und der Kontrollgruppe (Rauten) zu den verschiedenen Untersuchungszeitpunkten ${ }^{*}$ ( $p<0,01$ vs. Ruhe), ${ }^{* *}$ ( $p<0,01$ vs. Passiv), ${ }^{* * *}(p<0,001$ vs. Aktiv).

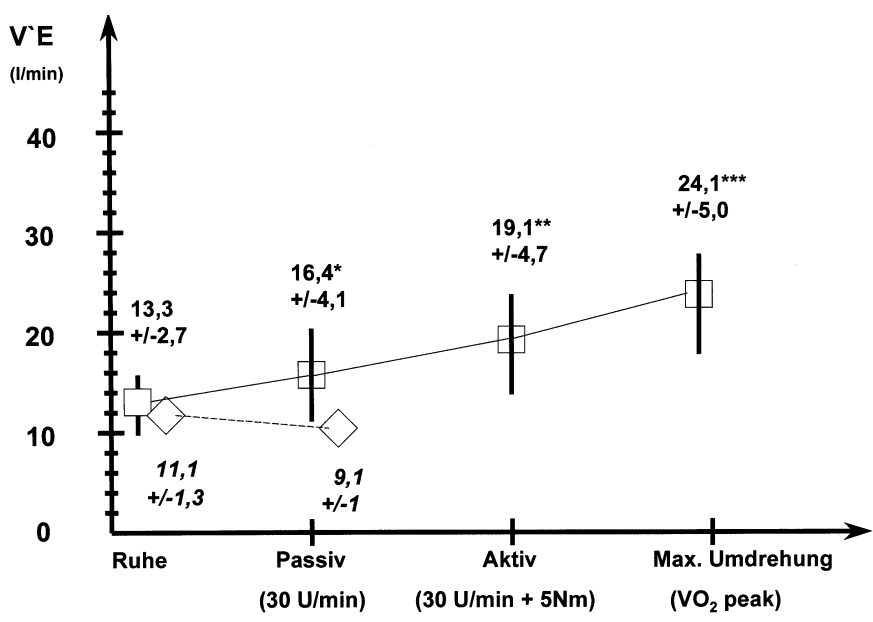

Abb. 3 Atemminutenvolumen VE der COB-Patienten (Rechtecke) und der Kontrollgruppe (Rauten) zu den verschiedenen Untersuchungszeitpunkten ${ }^{*}\left(p<0,01\right.$ vs. Ruhe) ${ }^{* *}$ ( $p<0,01$ vs. Passiv $),{ }^{* * *}(p<0,001$ vs. Aktiv).

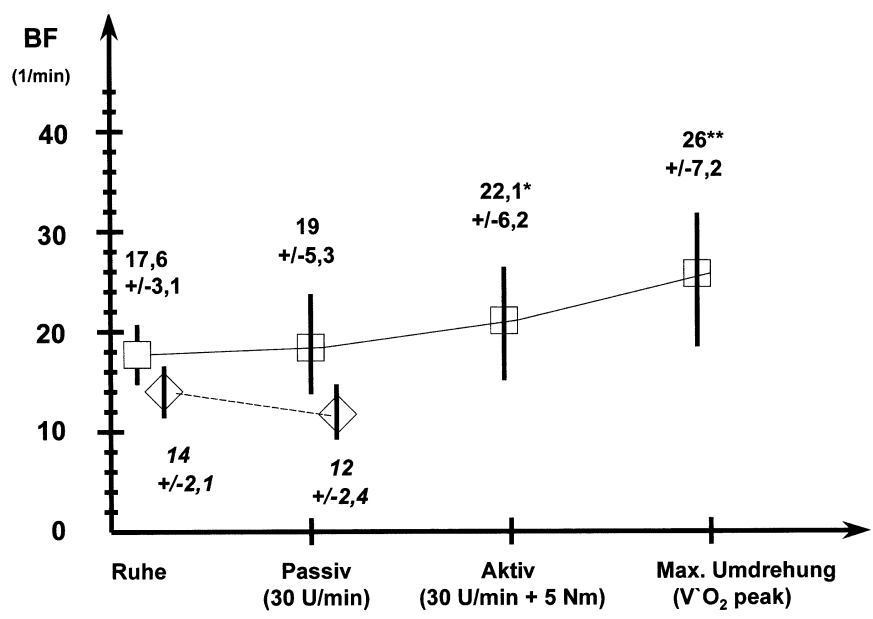

Abb. 4 Atemfrequenz BF der COB-Patienten (Rechtecke) und de Kontrollgruppe (Rauten) zu den verschiedenen Untersuchungszeitpunkten * ( $p=0,01$ vs. Passiv), ${ }^{* *}$ ( $p<0,01$ vs. Aktiv). 


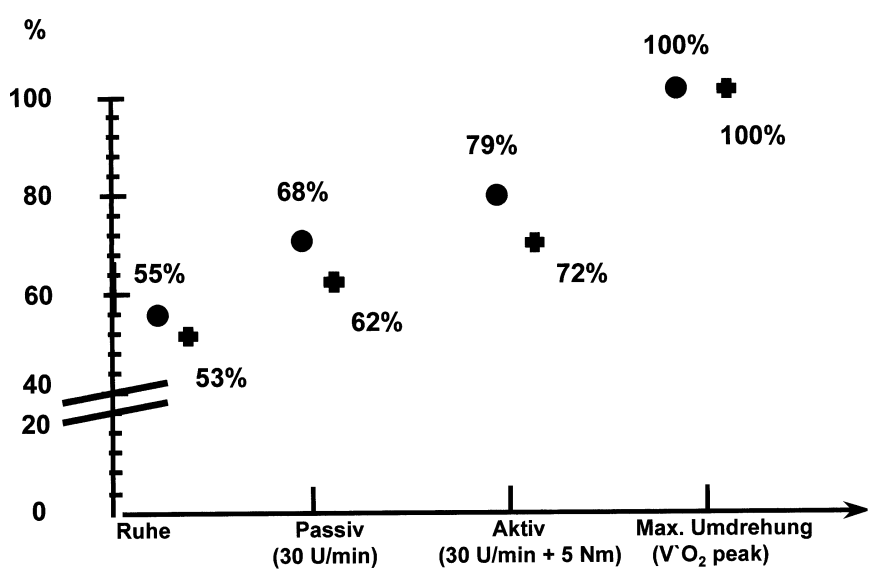

Abb. 5 Prozentualer Anteil der Sauerstoffaufnahme $\dot{\mathrm{VO}}_{2}$ (Kreise) und des Atemminutenvolumens $\dot{V} E$ (Kreuze) an der $\dot{V}_{2}$ peak bzw. am $\dot{V} E$ max zu den verschiedenen Untersuchungszeitpunkten bei den COB-Patienten.

\section{Diskussion}

Ziel der Arbeit war es, den Einfluss einer alleinigen passiven Tretbewegung und einer zusätzlichen aktiven Tretarbeit auf die Atmung bei weitgehend bettlägerigen Patienten mit einer schwergradigen chronisch-obstruktiven Bronchitis zu untersuchen. Dieses Patientengut ist aufgrund der Bettlägerigkeit und der erheblichen Muskelatrophie mit einer herkömmlichen Spiroergometrie bzw. einem 6-Minuten-Gehtest nicht zu belasten und konnte daher bislang einem an der individuellen maximalen Leistungsfähigkeit orientierten Ergometertraining nicht zugeführt werden. Mit sog. Passiv-Ergometern besteht die Möglichkeit, diese Patienten im Bett nach einer Phase rein passiver Bewegungsübungen zusätzlich aktiv gegen definierte Widerstände antreten zu lassen. Der Einfluss auf atemphysiologische Parameter wurde in diesem Zusammenhang bislang nicht untersucht.

Patienten mit einer schwergradigen $\mathrm{COB}$ weisen eine deutlich reduzierte maximale Belastbarkeit bzw. Sauerstoffaufnahme $\left(\mathrm{VO}_{2}\right.$ peak) auf. Casaburi u. Mitarb. dokumentierten bei ihrem Patientengut $\left(\mathrm{FEV}_{1} 0,93 \pm 0,27 \mathrm{l}\right)$ eine $\mathrm{VO}_{2}$ peak von $860 \mathrm{ml} / \mathrm{min}$ und ein maximales Atemminutenvolumen (VE max) von 33 1/min [12]. Andere Untersucher konnten diese Ergebnisse bestätigen [13 - 15]. Die von uns untersuchten COB-Patienten lagen mit ihrer $\dot{V}_{2}$ peak bzw. dem $\dot{V E}$ max noch deutlich unter diesen Werten, was vermutlich damit zu erklären ist, dass unsere Patienten weitgehend bettlägerig waren und zum Teil selbst einer Bodyplethysmographie nicht unterzogen werden konnten, während in den anderen Studien noch mobile Patienten untersucht wurden. Untersuchungen von Yoshikawa u. Mitarb. belegten, dass gerade COB-Patienten mit einer schwergradigen funktionellen Einschränkung eine disproportionale Reduktion der Muskelmasse der unteren Extremitäten aufweisen und diese zur früheren Belastungslimitation beiträgt [16]. Die Ausprägung der funktionellen Einschränkung zeigte sich bei unseren Patienten auch daran, dass sie in Ruhe bereits $53 \%$ ihres $\mathrm{VO}_{2}$ peak und $55 \%$ ihrer $\mathrm{VE}$ max leisteten.
Auffällig war, dass es bei den COB-Patienten in unserer Studie im Gegensatz zu den Gesunden zu einem Anstieg von Atemfrequenz, Atemminutenvolumen und Sauerstoffaufnahme alleine bei passiver Bewegung mit $30 \mathrm{U} / \mathrm{min}$ kam und diese immerhin ein Ausmaß von $62 \%$ des $\dot{V O}_{2}$ peak und $68 \%$ des $\dot{V}$ max dieser Patienten erreichte. Die belastungsinduzierte Hyperpnoe wird mit einer Reaktion auf afferente Stimuli aus Propriorezeptoren bzw. Muskelspindeln der Arbeitsmuskulatur erklärt [17], wobei jedoch tierexperimentelle Untersuchungen die Bedeutung dieser Rezeptoren eher infrage und kardiovaskuläre bzw. hämodynamische Einflüsse in den Vordergrund stellten [18,19]. Offenbar ist jedoch eine alleinige passive Bewegung bei Gesunden nicht in der Lage, einen Anstieg der Sauerstoffaufnahme zu erreichen, während diese bei den schwerst kompromittierten COB-Patienten - möglicherweise doch rezeptorvermittelt oder über einen vermehrten Rückfluss desaturierten Blutes aus der Muskulatur [17] - bereits eine vermehrte Ventilation induziert. Ähnliche Beobachtungen machten Sala u. Mitarb. sowie Nery u. Mitarb., die bei Patienten mit COB unter submaximaler (aktiver) Belastung eine signifikant höhere Sauerstoffaufnahme und ein signifikant größeres Atemminutenvolumen als bei Gesunden fanden $[20,21]$.

Empfohlen wird für COB-Patienten ein körperliches Training mit 60-80\% der maximalen Belastung zur Steigerung ihrer Belastbarkeit [8]. Bei den von uns untersuchten COB-Patienten ließen sich durch die zusätzliche aktive Mitarbeit die $\mathrm{VO}_{2}$ auf $72 \%$ des $\grave{V O}_{2}$ peak und das $\grave{V E}$ auf $79 \%$ des $\dot{V}$ max steigern.

Der Nachteil des von uns benutzten Passiv-Ergometers ist jedoch, dass die aktuelle Wattzahl nicht direkt angegeben werden kann, so dass eine Quantifizierung der Trainingsbelastung nur über die Umdrehungszahl bei konstantem Tretwiderstand möglich ist. Hier wäre in einem nächsten Schritt eine „Eichkurve“ zur Darstellung des Zusammenhangs zwischen Wattzahl, $\mathrm{O}_{2}$-Aufnahme und Umdrehungszahl zu erstellen. Damit ließe sich auch für bettlägerige COB-Patienten ein Trainingsprogramm entwerfen und eine Steigerung der Belastbarkeit objektivieren.

Zusammenfassend konnten wir feststellen, dass bettlägerige Patienten mit einer schwergradigen COB bereits in Ruhe 55\% ihres $\mathrm{VO}_{2}$ peak aufnehmen und schon unter alleiniger passiver Bewegung mittels Bett-Ergometer im Gegensatz zu Gesunden eine signifikante Steigerung der Sauerstoffaufnahme und des Atemminutenvolumens aufweisen, die bei zusätzlicher aktiver Mitarbeit annähernd 75\% ihrer maximalen Belastbarkeit erreichen. Mit dem Passiv-Ergometer kann somit auch für dieses Patientenklientel ein körperliches Training durchgeführt werden. 
${ }^{1}$ Wasserman K, Whipp BJ. Exercise physiology in health and disease. Am Rev Respir Dis 1975; 112: 219-249

${ }^{2}$ Rühle KH. Spiroergometrische Kenngrößen bei Gesunden und Patienten mit verschiedenen Lungenerkrankungen. In: Rühle KH. Praxisleitfaden der Spiroergoemtrie Berlin, Köln: Kohlhammer, 2001: 33-42

${ }^{3}$ Ullmer E, Solèr M, Hamm H, Perruchoud AP. Pathogenese, Diagnostik und Therapie der COPD. Pneumologie 2000; 54: 123-132

${ }^{4}$ British Thoracic Society Statement. COPD: summary of guidelines. Thorax 1997; 52 (Suppl 5): S2 - 21

${ }^{5}$ Gosselink R, Troosters T, Decramer M. Exercise training in COPD patient: the basic questions. Eur Respir J 1997; 10: 2884-2891

${ }^{6}$ Neder JA, Jones PW, Nery LE, Whipp BJ. Determinants of the exercise endurance capacity in patients with chronic obstructive pulmonary disease. The power-duration relationship. Am J Respir Crit Care Med 2000; 162(2Pt1): 497-504

${ }^{7}$ Kirsten D, Taube C, Lehnigk B, Jörres R, Magnussen H. The effect of short-term exercise training in patients with severe COPD. Am Rev Respir Critical Care Med 1995; 151: A685

${ }^{8}$ Worth H, Meyer A, Folgering H, Kirsten D, Lecheler J, Magnussen H, Pleyer K, Schmidt S, Schmitz M, Taube K, Wettengel R. Empfehlungen der Deutschen Atemwegsliga zum Sport und körperlichen Training bei Patienten mit obstruktiven Atemwegserkrankungen. Pneumologie 2000; 54: $61-67$

${ }^{9}$ Casaburi R, Patessio A, Ioli F, Zanaboni S, Donner CF, Wasserman K. Reductions in exercise lactic acidosis and ventilation as a result of exercise training in patients with obstructive lung disease. Am Rev Respir Dis 1991; 143: 9-18

${ }^{10}$ Keyser RE, Rodgers MM, Gardner ER, Russell PJ. Oxygen uptake during peak graded exercise and single-stage fatigue tests of wheelchair propulsion in manual wheelchair users and the able-bodied. Arch Phys Med Rehabil 1999; 80(10): 1288-1292

${ }^{11}$ ATS Statement. Standards for the diagnosis and care of patients with chronic obstructive pulmonary disease. Am J Respir Crit Care Med 1995; 152: S77-120
${ }^{12}$ Casaburi R, Porszasz J, Burns MR, Carithers ER, Chang RS, Cooper CB. Physiologic benefits of exercise training in rehabilitation of patients with severe chronic obstructive pulmonary disease. Am J Respir Crit Care Med 1997; 155(5): 1541 - 1551

13 Oelberg DA, Medoff BD, Markowitz DH, Pappagianopoulos PP, Ginns LC, Systrom DM. Systemic oxygen extraction during incremental exercise in patients with severe chronic obstructive pulmonary disease. Eur J Appl Physiol Occup Physiol 1998; 78(3): 201 - 207

${ }^{14}$ Gallagher CG. Exercise limitation and clinical exercise testing in chronic obstructive pulmonary disease. Clinics in Chest Medicine 1994; 15: $305-326$

${ }^{15}$ Leuppi JD, Zenhäusern R, Schwarz F, Frey WO, Villiger B. Bedeutung der Trainingsintensität für die Verbesserung der Ausdauerleistungsfähigkeit bei Patienten mit chronischen obstruktiven Lungenerkrankungen. Dtsch Med Wschr 1998; 123: 174-178

16 Yoshikawa M, Yoneda T, Takenada H, Fukuoka A, Okamoto Y, Narita N, Nezu K. Distribution of muscle mass and maximal exercise performance in patients with COPD. Chest 2001; 119: 93-98

17 Wasserman K, Hansen JE, Sue DY, Casaburi R, Whipp BJ. Physiology of exercise. In: Wasserman K, Hansen JE, Sue DY, Casaburi R, Whipp BJ. Principles of exercise testing and interpretation. Philadelphia: Lippincott Williams \& Wilkins, 1999: 10-61

${ }^{18}$ Hornbein TF, Sorenson SC, Parks CR. Role of muscle spindles in lower extremities in breathing during bicycle exercise. J Appl Physiol 1969; 27: $476-479$

${ }^{19}$ Hodgson HJF, Mathews PBC. The ineffectiveness of excitation of the primary endings of the muscle spindle by vibration as a respiratory stimulant in the decerebrate cat. J Physiol (London) 1968; 194: $555-563$

${ }^{20}$ Sala E, Roca J, Marrades RM, Alonso J, Gonzalez De Suso JM, Moreno A, Barbera JA, Nadal J, de Jover L, Rodriguez-Roisin R, Wagner PD. Effects of endurance training on skeletal muscle bioenergetics in chronic obstructive pulmonary disease. Am J Respir Crit Care Med 1999; 159(6): $1726-1734$

${ }^{21}$ Nery LE, Wasserman K, French W, Oren A, Davis JA. Contrasting cardiovascular and respiratory responses to exercise in mitral valve and chronic obstructive pulmonary disease. Chest 1983; 83: 446-453

\section{Atemwegsinfektion in Klinik und Praxis}

Lorenz, J. (Hrsg.)

UNI-MED Verlag AG, Bremen 2001. 145 Seiten, DM 79,80.

ISBN 3-89599-488-X

Respiratorische Infektionen haben eine enorme Häufigkeit. Ihre wichtige klinische Bedeutung gewinnen sie darüber hinaus über die Breite der verursachenden Agentien und die erheblich unterschiedlichen klinischen Schweregrade. Es ist das Verdienst von J. Lorenz, kompetente Autoren gewonnen zu haben, die in dem oben erwähnten Büchlein die gesamte Palette von Infektionen des Respirationstraktes abdecken. In dem formal ansprechenden und sehr gut strukturierten Buch gibt es ausgezeichnete Artikel über obere Atemwegsinfektionen, Tracheitiden sowie Bronchitiden. Dabei wird auch der Bogen zu speziellen Patientengruppen wie zum Beispiel Patienten mit zystischer Fibrose, Bronchiektasen-Krankheiten oder angeborenem Alpha-I-Antiproteinaseninhibitorenmangel gespannt. Man merkt der Veröffentlichung darüber hinaus an, dass die meisten Autoren über eine tiefgreifende Erfahrung auf dem gesamten Gebiet der Pneumologie verfügen. Insofern sind auch die über die rein infektiologische Problematik hinausgehenden Ausblicke von hohem Interesse.

Die zweite Hälfte des Buches behandelt die komplexe Thematik von Pneumonien. Hierbei werden in einzelnen Kapiteln ambulant erworbene Pneumonien, nosokomiale Pneumonien sowie Pneumonien bei Immun-Inkompetenz vorgestellt.
Ein abschließendes Kapitel behandelt die Antibiotika-Therapie bei Atemwegsinfektionen, organisiert nach verschiedenen Substanzgruppen. Von Interesse ist dabei, dass auch auf die Kosten der antibiotischen Intervention eingegangen wird. Dieser Gesichtspunkt spielt ja angesichts des kurativen Therapiecharakters und der kurzen Therapiedauer im ambulanten Bereich eine untergeordnete Rolle. Im stationären Bereich kann durch die überlegte Auswahl der antibiotisch wirksamen Substanzen jedoch eine erhebliche Menge Geld eingespart werden.

Das letzte Kapitel beschäftigt sich schließlich mit Impfungen, die im Rahmen der Prävention von Infektionen des Respirationstraktes eingesetzt werden können. Auch hier kann der Leser wertvolle Informationen gewinnen.

Insgesamt handelt es sich um eine gelungene Darstellung eines recht komplexen Gebietes. Die einzelnen Beiträge sind flüssig geschrieben und lesen sich gut. Wegen der guten grafischen Aufmachung eignet sich das Buch jedoch in meinen Augen insbesondere als Nachschlagewerk, das bei Bedarf zu konkreten Fragestellungen Auskunft geben kann.

T. Schaberg, Rotenburg 